[RAdiocarbon, Vol 23, No. 1, 1981, P 24-32]

\title{
US GEOLOGICAL SURVEY, DENVER, COLORADO RADIOCARBON DATES III
}

\author{
I C YANG, R L McAVOY, and R L EMERSON \\ US Geological Survey, Denver, Colorado 80225 \\ INTRODUCTION
}

This list contains the results of measurements made during 1978 and 1979. Ages were computed on the radiocarbon half-life of $5568 \pm 30$ years. Statistical errors quoted herein are $1 \sigma$ counting errors which include sample, background, and standard. The age limit reported is calculated on the basis of $3 \sigma$ activity. The $\delta^{13} \mathrm{C}$ values in table 1 were measured by Krueger Enterprises, Inc, Geochron Laboratories Division ${ }^{1}$ and calculated based on Craig's Peedee Belemnite (PDB) limestone standard (Craig, 1957). The total alkalinity as bicarbonate values reported in table 1 was determined using techniques described by Brown, Skougstad, and Fishman (1970). Unless otherwise stated, all samples were collected and submitted by personnel of the US Geological Survey.

Benzene was synthesized from precipitated barium carbonate (Noakes, Kim, and Akers, 1967). Since the publications of Schroder et al (1973) and Schroder, Emerson, and Beetem (1978), improvements in the counting techniques have been made (Yang and Emerson, 1979). Samples were counted in Teflon vials made of DuPont's polytetrafloro ethylene (PTFE) rod, similar to the designs of Calf and Polach (1974) and our low ${ }^{40} \mathrm{~K}$ glass vial previously used. This resulted in an increase in ${ }^{14} \mathrm{C}$ counting efficiency of 5 percent for $3 \mathrm{~mL}$ to $1 \mathrm{~mL}$ benzene, PCS (phase combining solvent) and 10 percent increase for $4 \mathrm{~mL}$ to $1 \mathrm{~mL}$ benzene, PCS counting solution relative to low ${ }^{40} \mathrm{~K}$ glass vial. No significant decrease in background count rate was observed for $3 \mathrm{~mL}$ to $1 \mathrm{~mL}$, benzene, PCS solution in Teflon vial compared to glass vial. However, for larger sample sizes ( $4 \mathrm{~mL}$ to $1 \mathrm{~mL}$ and $5 \mathrm{~mL}$ to $1 \mathrm{~mL}$ benzene, PCS), the background count rates in Teflon vials remained nearly the same as for $3 \mathrm{~mL}$ to $1 \mathrm{~mL}$ benzene, PCS, yet low ${ }^{40} \mathrm{~K}$ glass vial background count rate increased by approximately 1.25 counts per minute per milliliter of counting solution (Yang and Emerson, 1979).

\section{SAMPLE DESGRIPTIONS}

\section{A. Oregon}

DE-1. 01N/02E-24aad

$3100 \pm 160$

Sample coll Nov 17, 1977, from Multnomah Co $\left(45^{\circ} 33^{\prime} 36^{\prime \prime} \mathrm{N}, 122^{\circ}\right.$ $29^{\prime} 57^{\prime \prime} \mathrm{W}$ ) from depth $139.3 \mathrm{~m}$. Hole drilled to $149.4 \mathrm{~m}$. Alt of well head $8.1 \mathrm{~m}$ above msl. Static water level was $2.1 \mathrm{~m}$.

DE-2. 01N/02E-15cba

Sample coll Nov 3, 1977, from Multnomah Co $\left(45^{\circ} 34^{\prime} 07^{\prime \prime} \mathrm{N}, 122^{\circ}\right.$ $33^{\prime} 13^{\prime \prime}$ W) from depth $68.9 \mathrm{~m}$. Hole drilled to $68.9 \mathrm{~m}$. Alt of well head $5.3 \mathrm{~m}$ above msl. Static water level was $2.7 \mathrm{~m}$.

${ }^{1}$ The use of company and brand names in this paper is for identification only and does not imply endorsement by the US Geological Survey. 
DE-3. 01N/02E-15cba

$2600 \pm 210$

Sample coll Nov 9, 1977, from Multnomah Co $\left(45^{\circ} 34^{\prime} 22^{\prime \prime} \mathrm{N}, 122^{\circ}\right.$ $33^{\prime} 41^{\prime \prime} \mathrm{W}$ ) from depth $20.7 \mathrm{~m}$ to $67.1 \mathrm{~m}$. Hole drilled to $121.9 \mathrm{~m}$. Alt of well head $5.2 \mathrm{~m}$ above msl. Static water level was $2.7 \mathrm{~m}$.

DE-4. 01N/02E-15bea

$100 \pm 140$

Sample coll Nov 9, 1977, from Multnomah Co $\left(45^{\circ} 34^{\prime} 22^{\prime \prime} \mathrm{N}, 122^{\circ}\right.$ $33^{\prime} 13^{\prime \prime}$ W) from depth $74.4 \mathrm{~m}$ to $97.5 \mathrm{~m}$. Hole drilled to $100 \mathrm{~m}$. Alt of well head $7.7 \mathrm{~m}$ above msl. Static water level was $6.1 \mathrm{~m}$.

\section{DE-5. 01N/02E-24adc}

$3000 \pm 140$

Sample coll Dec 17, 1977, from Multnomah Co $\left(45^{\circ} 33^{\prime} 23^{\prime \prime} \mathrm{N}, 122^{\circ}\right.$ $29^{\prime} 57^{\prime \prime}$ W) from depth $76.5 \mathrm{~m}$ to $97.5 \mathrm{~m}$. Hole drilled to $97.5 \mathrm{~m}$. Alt of well head $5.2 \mathrm{~m}$ above msl. Static water level was $3 \mathrm{~m}$.

DE-6. 01N/03E-2lace

$<\mathbf{1 0 0}$

Sample coll Jan 26, 1977, from Multnomah Co $\left(45^{\circ} 33^{\prime} 26^{\prime \prime} \mathrm{N}, 122^{\circ}\right.$ $26^{\prime} 29^{\prime \prime}$ W) from depth $13.4 \mathrm{~m}$ to $39.6 \mathrm{~m}$. Hole drilled to $45.7 \mathrm{~m}$. Alt of well head $5.7 \mathrm{~m}$ above msl. Static water level was $2.1 \mathrm{~m}$.

\section{B. Utah}

DE-7.

Sample coll Aug 16, 1978, from Grand Co (38 $50^{\circ} 37^{\prime \prime} \mathrm{N}, 109^{\circ} 44^{\prime}$ $01^{\prime \prime} \mathrm{W}$ ) at depth 118.9 to $163.1 \mathrm{~m}$. Sample contained $\mathrm{H}_{2} \mathrm{~S}$.

DE-8. Well (-23-6) 6ace

$31,000 \pm 840$

Sample coll Nov 28, 1978, from Emery Co $\left(38^{\circ} 50^{\prime} 45^{\prime \prime} \mathrm{N}, 111^{\circ} 17^{\prime}\right.$ $18^{\prime \prime} \mathrm{W}$ ) at depth $219.5 \mathrm{~m}$.

DE-9. Well (D-22-6) 17abc

$27,700 \pm 850$

Sample coll Dec 14, 1978, from Emery Co $\left(38^{\circ} 54^{\prime} 26^{\prime \prime} \mathrm{N}, 111^{\circ} 16^{\prime}\right.$ $08^{\prime \prime} \mathrm{W}$ ) at depth $335.3 \mathrm{~m}$.

DE-10.

\section{Nevada}

Sample coll July 21, 1978, from Churchill Co $\left(39^{\circ} 29^{\prime} 19^{\prime \prime} \mathrm{N}, 118^{\circ}\right.$ $48^{\prime} 34^{\prime \prime} \mathrm{W}$ ) at depth $39.6 \mathrm{~m}$. Well pumped several days before sampling.

DE-11.

$9900 \pm 210$

Sample coll July 19, 1978 , from Churchill Co $\left(39^{\circ} 22^{\prime} 54^{\prime \prime} \mathrm{N}, 118^{\circ}\right.$ $43^{\prime} 18^{\prime \prime} \mathrm{W}$ ) at depth $36 \mathrm{~m}$. Well pumped $1500 \mathrm{~L}$ before sampling.

DE-12.

$5300 \pm 160$

Sample coll Aug 10, 1978, from Churchill Co $\left(39^{\circ} 28^{\circ} 39^{\prime \prime} \mathrm{N}, 118^{\circ}\right.$ $46^{\prime} 34^{\prime \prime} \mathrm{W}$ ) at depth $146.3 \mathrm{~m}$. Well pumped several hr before sampling.

DE-13.

$7600 \pm 150$

Sample coll Oct 6, 1978, from Churchill Co $\left(39^{\circ} 28^{\prime} 25^{\prime \prime} \mathrm{N}, 118^{\circ} 47^{\prime}\right.$ $08^{\prime \prime}$ W) at depth $153.9 \mathrm{~m}$. 
TABLE 1

Summary of $\delta \mathrm{C}^{13}$ and alkalinity for water sources

\begin{tabular}{|c|c|c|c|c|}
\hline $\begin{array}{l}\text { Sample } \\
\text { no. }\end{array}$ & $\begin{array}{l}\text { Coll } \\
\text { date }\end{array}$ & $\left(\begin{array}{l}\delta C^{13} \\
\% \text { PDB })\end{array}\right.$ & $\begin{array}{l}\text { Total alkalinity } \\
\text { as bicarbonate } \\
\text { (mg/L) }\end{array}$ & $\begin{array}{l}\text { Water source } \\
\text { by state }\end{array}$ \\
\hline$D E-1$ & $11 / 17 / 77$ & -20.0 & 78 & Oregon \\
\hline$D E-2$ & $11 / 3 / 77$ & -20.7 & 62 & 11 \\
\hline$D E-3$ & $11 / 9 / 77$ & -19.8 & 76 & $"$ \\
\hline $\mathrm{DE}-4$ & $11 / 9 / 77$ & -11.5 & 419 & $"$ \\
\hline $\mathrm{DE}-5$ & $12 / 17 / 77$ & -20.4 & 85 & $"$ \\
\hline $\mathrm{DE}-6$ & $1 / 26 / 78$ & -20.8 & 61 & $"$ \\
\hline $\mathrm{DE}-7$ & $8 / 16 / 78$ & -19.4 & -- & Utah \\
\hline $\mathrm{DE}-8$ & $11 / 28 / 78$ & -12.6 & -- & " \\
\hline$D E-9$ & $12 / 14 / 78$ & -10.0 & -- & $"$ \\
\hline$D E-10$ & $7 / 21 / 78$ & -- & -- & Nevada \\
\hline$D E-11$ & $7 / 19 / 78$ & -- & -- & $"$ \\
\hline$D E-12$ & $8 / 10 / 78$ & -- & -- & $"$ \\
\hline $\mathrm{DE}-13$ & $10 / 6 / 78$ & -- & -- & $"$ \\
\hline$D E-14$ & $2 / 8 / 78$ & -- & -- & $"$ \\
\hline$D E-15$ & $6 / 21 / 79$ & - & 268 & $"$ \\
\hline$D E-16$ & $6 / 20 / 79$ & -- & -- & $"$ \\
\hline$D E-17$ & $6 / 22 / 79$ & -- & -- & $"$ \\
\hline$D E-18$ & $6 / 20 / 79$ & -- & 192 & $"$ \\
\hline$D E-19$ & $6 / 19 / 79$ & - & 374 & $"$ \\
\hline$D E-20$ & $6 / 25 / 79$ & -- & -- & $"$ \\
\hline$D E-21$ & $6 / 26 / 79$ & -- & 180 & $"$ \\
\hline$D E-22$ & $7 / 2 / 79$ & -14.1 & -- & South Dakota \\
\hline$D E-23$ & $7 / 3 / 79$ & -11.4 & -- & " \\
\hline$D E-24$ & $7 / 17 / 79$ & -12.2 & -- & $"$ \\
\hline$D E-25$ & $7 / 16 / 79$ & -11.7 & -- & $"$ \\
\hline$D E-26$ & $7 / 26 / 79$ & -7.7 & 179 & $"$ \\
\hline$D E-27$ & $7 / 26 / 79$ & -8.2 & 175 & $"$ \\
\hline$D E-28$ & $7 / 27 / 79$ & -8.5 & 175 & $"$ \\
\hline$D E-29$ & $7 / 24 / 79$ & -5.2 & 182 & $"$ \\
\hline$D E-30$ & $7 / 19 / 79$ & -11.0 & 390 & $"$ \\
\hline$D E-31$ & $7 / 30 / 79$ & -8.7 & 195 & $"$ \\
\hline$D E-32$ & $7 / 29 / 79$ & -6.8 & 182 & $"$ \\
\hline$D E-33$ & $7 / 31 / 79$ & -8.0 & 175 & $"$ \\
\hline$D E-34$ & $7 / 31 / 79$ & -13.2 & 378 & $"$ \\
\hline$D E-35$ & $7 / 29 / 79$ & -7.7 & 190 & $"$ \\
\hline$D E-36$ & $7 / 28 / 79$ & -7.3 & 182 & $"$ \\
\hline$D E-37$ & $8 / 6 / 79$ & -20.2 & 633 & $"$ \\
\hline $\mathrm{DE}-38$ & $8 / 5 / 79$ & -11.4 & 64 & $"$ \\
\hline$D E-39$ & $8 / 4 / 79$ & -8.3 & 163 & $"$ \\
\hline$D E-40$ & $8 / 7 / 79$ & -26.2 & 864 & $"$ \\
\hline$D E-41$ & $8 / 6 / 79$ & -19.5 & 980 & $"$ \\
\hline$D E-42$ & $8 / 6 / 79$ & -14.0 & 242 & $"$ \\
\hline$D E-43$ & $8 / 4 / 79$ & -9.9 & 204 & $"$ \\
\hline $\mathrm{DE}-44$ & $8 / 1 / 79$ & -15.8 & 446 & $"$ \\
\hline$D E-45$ & $8 / 3 / 79$ & -11.0 & 173 & $"$ \\
\hline$D E-46$ & $8 / 2 / 79$ & -10.2 & 211 & $"$ \\
\hline$D E-47$ & $8 / 1 / 79$ & -14.0 & 431 & $"$ \\
\hline$D E-48$ & $8 / 21 / 79$ & -24.2 & - & $"$ \\
\hline$D E-49$ & $8 / 29 / 79$ & -10.6 & - & $"$ \\
\hline$D E-50$ & $8 / 30 / 79$ & -13.4 & -- & $"$ \\
\hline$D E-51$ & $9 / 7 / 79$ & -11.1 & -- & $"$ \\
\hline $\mathrm{DE}-52$ & $9 / 6 / 79$ & -15.9 & -- & $"$ \\
\hline$D E-53$ & $8 / 9 / 79$ & -10.2 & 214 & Wyoming \\
\hline $\mathrm{DE}-54$ & $8 / 11 / 79$ & -7.0 & 235 & " \\
\hline$D E-55$ & $8 / 6 / 79$ & -9.6 & 213 & $"$ \\
\hline$D E-56$ & $8 / 6 / 79$ & -11.6 & 225 & $"$ \\
\hline$D E-57$ & $8 / 12 / 79$ & -9.4 & 365 & $"$ \\
\hline$D E-58$ & $8 / 12 / 79$ & -14.4 & 418 & $"$ \\
\hline$D E-59$ & $8 / 9 / 79$ & -16.0 & 107 & 4 \\
\hline$D E-60$ & $8 / 12 / 79$ & -13.3 & 231 & $"$ \\
\hline$D E-61$ & $8 / 8 / 79$ & -9.2 & 195 & $"$ \\
\hline $\mathrm{DE}-62$ & $8 / 9 / 79$ & -14.0 & 214 & $"$ \\
\hline$D E-63$ & $8 / 9 / 79$ & -7.9 & 320 & $"$ \\
\hline$D E-64$ & $8 / 11 / 79$ & -10.1 & 244 & $"$ \\
\hline$D E-65$ & $8 / 7 / 79$ & -9.3 & 210 & $"$ \\
\hline$D E-66$ & $8 / 7 / 79$ & -16.1 & 58 & $"$ \\
\hline$D E-67$ & $8 / 4 / 79$ & -16.6 & 220 & $"$ \\
\hline$D E-68$ & $8 / 4 / 79$ & -11.6 & 190 & $"$ \\
\hline
\end{tabular}


DE-14.

$14,100 \pm 400$

Sample coll Feb 8, 1978, from Churchill Co $\left(39^{\circ} 33^{\prime} 40^{\prime \prime} \mathrm{N}, 118^{\circ}\right.$ $43^{\prime} 19^{\prime \prime} \mathrm{W}$ ) at depth $79.2 \mathrm{~m}$. Sample coll $4 \mathrm{hr}$ after starting pumping.

\section{DE-15. Well 10S/47-30dec}

$8400 \pm 140$

Sample coll June 21, 1979, from Windmill Ranch $13 \mathrm{~km} \mathrm{~N}$ of Beatty in Oasis Valley, Nye Co $\left(37^{\circ} 01^{\prime} 60^{\prime \prime} \mathrm{N}, 116^{\circ} 45^{\prime} 30^{\prime \prime} \mathrm{W}\right)$. Hole drilled to $36.9 \mathrm{~m}$ and cased to $19.8 \mathrm{~m}$ below land surface datum. Alt of well head $1179.6 \mathrm{~m}$ above msl. Static water level was $5.5 \mathrm{~m}$ below land surface datum. Pump yielded 190Lpm.

DE-16. Spring 10S/47-33aab

Sample coll June 20, 1979, from spring in Oasis Valley, Nye Co $\left(37^{\circ} 01^{\prime} 49^{\prime \prime} \mathrm{N}, 116^{\circ} 43^{\prime} 08^{\prime \prime} \mathrm{W}\right)$. Ditch along base of steep volcanic slope intersects seeps from fractured volcanic rock and channels water to a reservoir. Alt of reservoir $1182.6 \mathrm{~m}$ above msl.

DE-17. Water Supply Well J-13

$9900 \pm 130$

Sample coll June 22, 1979, from Nevada Test Site, Nye Co $\left(36^{\circ} 48^{\prime}\right.$ $\left.29^{\prime \prime} \mathrm{N}, 116^{\circ} 23^{\prime} 40^{\prime \prime} \mathrm{W}\right)$. Hole drilled to $1063.1 \mathrm{~m}$ and cased to $1031.7 \mathrm{~m}$ below land surface datum. Alt of well head $1011.3 \mathrm{~m}$ above msl. Static water level was $282.9 \mathrm{~m}$ below land surface datum. Casing perforated 303.5 to $422.5 \mathrm{~m}$ and 819.9 to $1009.5 \mathrm{~m}$. Pump discharged 2600L $\mathrm{pm}$.

\section{DE-18. Spring 10S/47-14bab}

$7600 \pm 100$

Sample coll June 20, 1979, from Fleur de Lis Ranch, Oasis Valley, Nye Co $\left(37^{\circ} 04^{\prime} 27^{\prime \prime} \mathrm{N}, 116^{\circ} 41^{\prime} 24^{\prime \prime} \mathrm{W}\right)$ from depth $1.8 \mathrm{~m}$ below land surface datum. Alt of spring $1213.7 \mathrm{~m}$ above msl.

DE-19. Well 12S/47-7dbd

$3500 \pm 60$

Sample coll June 19, 1979, from Beatty Municipal Well \#2, Oasis Valley, Nye Co $\left(36^{\circ} 54^{\prime} 18^{\prime \prime} \mathrm{N}, 116^{\circ} 45^{\prime} 24^{\prime \prime} \mathrm{W}\right)$ from depth 54.9 to $91.4 \mathrm{~m}$. Hole drilled to $91.4 \mathrm{~m}$ and cased to $54.9 \mathrm{~m}$ below land surface datum. Pump yielded 870L $\mathrm{Lm}$.

DE-20. Well 10S/47-14bab

$21,500 \pm 330$

Sample coll June 25, 1979, from Coffer's Well, Oasis Valley, Nye Co $\left(37^{\circ} 04^{\prime} 27^{\prime \prime} \mathrm{N}, 116^{\circ} 41^{\prime} 24^{\prime \prime} \mathrm{W}\right)$. A $2.4 \mathrm{~m}$ well beside a reservoir was equipped with a centrifugal pump from which water was obtained for analysis. Springs were located in bottom of reservoir. Alt of well head is $1213.7 \mathrm{~m}$ above msl. Pump yielded $1325 \mathrm{Lpm}$.

DE-21. Well 11S/47-27cha

$9300 \pm 120$

Sample coll June 26, 1979, from Marvin's Well, Oasis Valley, Nye Co $\left(36^{\circ} 56^{\prime} 57^{\prime \prime} \mathrm{N}, 116^{\circ} 42^{\prime} 46^{\prime \prime}\right.$ W) $5.6 \mathrm{~km} \mathrm{~N}$ of Beatty, Nevada at depth $10.6 \mathrm{~m}$. Hole drilled to $16.8 \mathrm{~m}$ and cased to $15.2 \mathrm{~m}$ below land surface datum. Alt of well head $1060.7 \mathrm{~m}$ above msl. Static water level was $9.1 \mathrm{~m}$ below land surface datum. Pump yielded 76L pm. 


\section{South Dakota}

DE-22. Well 7S/1E-15acda (TVA Lakota)

$18,300 \pm 330$

Sample coll July 2, 1979, from Fall River Co $\left(43^{\circ} 26^{\prime} 38^{\prime \prime} \mathrm{N}, 103^{\circ}\right.$ $58^{\prime} 52^{\prime \prime}$ W) at depth $182 \mathrm{~m}$.

DE-23. Well 11N/1E-17deac $>37,500$

Sampled coll July 3, 1979, from Russ Carver Well \#2, Butte Co $\left(44^{\circ} 54^{\prime} 41^{\prime \prime} \mathrm{N}, 104^{\circ} 01^{\prime} 24^{\prime \prime} \mathrm{W}\right)$ at depth $640 \mathrm{~m}$.

DE-24. Well 5N/9E-34cchd

Sample coll July 17,1979 , from Meade Co $\left(44^{\circ} 20^{\prime} 47^{\prime \prime} \mathrm{N}, 103^{\circ} 01^{\prime}\right.$ $18^{\prime \prime} \mathrm{W}$ ) at depth $722.4 \mathrm{~m}$.

\section{DE-25. Well 8N/2E-14de}

$8100 \pm 140$

Sample coll July 16, 1979, from Butte Co $\left(44^{\circ} 39^{\prime} 05^{\prime \prime} \mathrm{N}, 103^{\circ} 50^{\prime}\right.$ $12 "$ W) at depth 131.1m.

DE-26. Well 104N/61W-36ddaa

$>26,200$

Sample coll July 26, 1979, from Richard Renken Well $\left(43^{\circ} 45^{\prime} 55^{\prime \prime}\right.$ $\left.\mathrm{N}, 98^{\circ} 05^{\prime} 12^{\prime \prime} \mathrm{W}\right)$ at depth $103.6 \mathrm{~m}$. Static water level was $6.5 \mathrm{~m}$ below land surface datum.

DE-27. Well 105N/58W-31bacc

$>\mathbf{3 0 , 6 0 0}$

Sample coll July 26, 1979 , from Miner Co $\left(43^{\circ} 51^{\prime} 42^{\prime \prime} \mathrm{N}, 97^{\circ} 50^{\prime}\right.$ $39^{\prime \prime}$ W) at depth $192 \mathrm{~m}$; flow rate $144 \mathrm{Lpm}$. Static water level was $10.8 \mathrm{~m}$ below land surface datum.

DE-28. Well 113N/61W-cbbb

Sample coll July 27, 1979 from Beadle Co $\left(44^{\circ} 34^{\prime} 12^{\prime \prime}\right.$ N, $98^{\circ} 09^{\prime}$ $34^{\prime \prime}$ W) at depth $274.3 \mathrm{~m}$; flow rate $151 \mathrm{Lpm}$.

DE-29. Well 109N/70W-babd

$>\mathbf{3 0 , 7 0 0}$

Sample coll July 24, 1979, from Hand Co $\left(44^{\circ} 16^{\prime} 53^{\prime \prime}\right.$ N, $99^{\circ} 15^{\prime}$ $33^{\prime \prime} \mathrm{W}$ ) at depth $592.8 \mathrm{~m}$; flow rate $151 \mathrm{Lpm}$. Alt of well head $60.5 \mathrm{~m}$ above msl.

DE-30. Well 1N/15E-36adcc

$>40,300$

Sample coll July 19, 1979 , from Pennington Co $\left(44^{\circ} 00^{\prime} 06^{\prime \prime} \mathrm{N}, 102^{\circ}\right.$ $15^{\prime} 04^{\prime \prime} \mathrm{W}$ ) at depth $378.1 \mathrm{~m}$.

\section{DE-31. Well 123N/64W-27ddaa}

Sample coll July 30, 1979, from Brown Co $\left(45^{\circ} 26^{\prime} 40^{\prime \prime} \mathrm{N}, 98^{\circ} 30^{\prime}\right.$ $50^{\prime \prime} \mathrm{W}$ ) at depth $342.9 \mathrm{~m}$; flow rate $57 \mathrm{Lpm}$.

DE-32. Well 128N/66W-8abbc

Sample coll July 29, 1979, from McPherson Co $\left(45^{\circ} 55^{\prime} 27^{\prime \prime} \mathrm{N}, 98^{\circ}\right.$ $49^{\prime} 03^{\prime \prime} \mathrm{W}$ ) at depth $581.2 \mathrm{~m}$; flow rate $227 \mathrm{Lpm}$.

DE-33. Well 122N/62W-23dadd

Sample coll July 31 , 1979 , from Brown Co $\left(45^{\circ} 21^{\prime} 44^{\prime \prime}\right.$ N, $98^{\circ} 14^{\prime}$ $51^{\prime \prime}$ W) at depth $349.9 \mathrm{~m}$; flow rate $34 \mathrm{Lpm}$. 
Sample coll July 31, 1979, from Marshall Co $\left(45^{\circ} 40^{\prime} 27^{\prime \prime}\right.$ N, $97^{\circ} 43^{\prime}$ $22^{\prime \prime} \mathrm{W}$ ) at depth $378.6 \mathrm{~m}$; flow rate $11.4 \mathrm{~L}$ pm. Static water level was $30.5 \mathrm{~m}$ below land surface datum.

\section{DE-35. Well 128N/67W-35bbcd2}

$31,900 \pm 1700$

Sample coll July 29, 1979 , from McPherson Co $\left(45^{\circ} 51^{\prime} 48^{\prime \prime} \mathrm{N}, 98^{\circ}\right.$ $52^{\prime} 53^{\prime \prime}$ W) at depth $515.4 \mathrm{~m}$; flow rate 227Lpm.

\section{DE-36. Well 123N/65W-22aad}

$29,000 \pm 1400$

Sample coll July 28, 1979, from Brown Co $\left(45^{\circ} 27^{\prime} 29^{\prime \prime} \mathrm{N}, 98^{\circ} 38^{\prime}\right.$ $24^{\prime \prime} \mathrm{W}$ ) at depth $409.3 \mathrm{~m}$; flow rate $189 \mathrm{Lpm}$. Static water level was $100.6 \mathrm{~m}$ below land surface datum.

\section{DE-37. Well 1N/24E-9cadd}

Sample coll Aug 6, 1979, from Haakon Co $\left(44^{\circ} 03^{\prime} 25^{\prime \prime} \mathrm{N}, 101^{\circ} 14^{\prime}\right.$ $13^{\prime \prime} \mathrm{W}$ ) at depth $722.4 \mathrm{~m}$; flow rate $568 \mathrm{Lpm}$. Static water level was $70.4 \mathrm{~m}$ below land surface datum.

DE-38. Well 99N/74W-1eded $>\mathbf{2 3 , 0 0 0}$

Sample coll Aug 5, 1979, from Tripp Co $\left(43^{\circ} 24^{\prime} 57^{\prime \prime}\right.$ N, $99^{\circ} 32^{\prime} 52^{\prime \prime}$ W) at depth $457.2 \mathrm{~m}$; flow rate $1.9 \mathrm{Lpm}$.

DE-39. Well 98N/70W-11c $29,900 \pm 1400$

Sample coll Aug 4, 1979, from Gregory Co $\left(43^{\circ} 19^{\prime} 45^{\prime \prime}\right.$ N, $99^{\circ} 05^{\prime}$ $20^{\prime \prime} \mathrm{W}$ ) at depth $259.1 \mathrm{~m}$; flow rate $114 \mathrm{Lpm}$. Static water level was $16.2 \mathrm{~m}$ below land surface datum.

DE-40. Well 6N/24E-03baaa

Sample coll Aug 7, 1979, from Haakon Co $\left(44^{\circ} 30^{\prime} 40^{\prime \prime} \mathrm{N}, 101^{\circ} 12^{\prime}\right.$ $30^{\prime \prime} \mathrm{W}$ ) at depth $792.5 \mathrm{~m}$; flow rate $15 \mathrm{Lpm}$.

DE-41. Well 1N/20E-24bcab

Sample coll Aug 6, 1979, from Haakon Co $\left(44^{\circ} 02^{\prime} 02^{\prime \prime} \mathrm{N}, 101^{\circ} 39^{\prime \prime}\right.$ $40^{\prime \prime} \mathrm{W}$ ) at depth $780.9 \mathrm{~m}$; flow rate $379 \mathrm{Lpm}$.

DE-42. Well 2S/22E-28bad

Sample coll Aug 6, 1979, from Jackson Co $\left(43^{\circ} 49^{\prime} 55^{\prime \prime} \mathrm{N}, 101^{\circ} 31^{\prime}\right.$ $20^{\prime \prime} \mathrm{W}$ ) at depth $832.1 \mathrm{~m}$; flow rate $946 \mathrm{Lpm}$.

\section{DE-43. Well 96N/63W-08cda}

Sample coll Aug 4, 1979, from Charles Mix Co $\left(43^{\circ} 08^{\prime} 26^{\prime \prime} \mathrm{N}, 98^{\circ}\right.$ $19^{\prime} 06^{\prime \prime} \mathrm{W}$ ) at depth $204.8 \mathrm{~m}$; flow rate $22.7 \mathrm{Lpm}$. Static water level was $10 \mathrm{~m}$ below land surface datum.

DE-44. Well 107N/49W-14abbc

Sample coll Aug 1, 1979, from Moody Co $\left(44^{\circ} 04^{\prime} 41^{\prime \prime}\right.$ N, $96^{\circ} 40^{\prime}$ $36^{\prime \prime} \mathrm{W}$ ) at depth $143.3 \mathrm{~m}$; flow rate $5.7 \mathrm{Lpm}$. 
DE-45. Well 93N/55W-4bbc

Sample coll Aug 3, 1979, from Yankton Co $\left(42^{\circ} 54^{\prime} 40^{\prime \prime} \mathrm{N}, 97^{\circ} 21^{\prime}\right.$ $25^{\prime \prime} \mathrm{W}$ ) at depth $124.1 \mathrm{~m}$; flow rate $38 \mathrm{Lpm}$.

DE-46. Well 91N/49W-19bcca

Sample coll Aug 2, 1979, from Union Co $\left(42^{\circ} 41^{\prime} 13^{\prime \prime} \mathrm{N}, 96^{\circ} 41^{\prime} 13^{\prime \prime}\right.$ W) at depth $99.1 \mathrm{~m}$; flow rate $833 \mathrm{Lpm}$.

DE-47. 127N/49W-29bbbc

Sample coll Aug 1, 1979, from Perkins Co $\left(45^{\circ} 45^{\prime} 35^{\prime \prime}\right.$ N, $96^{\circ} 49^{\prime} 58^{\prime \prime}$ W) at depth $161.5 \mathrm{~m}$; flow rate $9.8 \mathrm{Lpm}$. Static water level was $3.86 \mathrm{~m}$ below land surface datum.

DE-48. Well 5N/17E-21aace

Sample coll Aug 21, 1979, from Pennington Co $\left(44^{\circ} 23^{\prime} 00^{\prime \prime} \mathrm{N}\right.$, $102^{\circ} 03^{\prime} 53^{\prime \prime} \mathrm{W}$ ) at depth $938.8 \mathrm{~m}$.

DE-49. Well 8S/6E-14cbba

$17,400 \pm 230$

Sample coll Aug 29, 1979, from Fall River Co $\left(43^{\circ} 21^{\prime} 13^{\prime \prime} \mathrm{N}, 103^{\circ}\right.$ $\left.22^{\prime} 48^{\prime \prime} \mathrm{W}\right)$ at depth $609 \mathrm{~m}$.

DE-50. Well 11S/2E-21de

Sample coll Aug 30, 1979, from Fall River Co $\left(43^{\circ} 04^{\prime} 16^{\prime \prime} \mathrm{N}, 103^{\circ}\right.$ $52^{\prime} 41^{\prime \prime} \mathrm{W}$ ) at depth $731.5 \mathrm{~m}$.

DE-51. Well 2S/13E-14acad

$>36,600$

Sample coll Sept 7, 1979, from Pennington Co $\left(43^{\circ} 52^{\prime} 27^{\prime \prime} \mathrm{N}, 102^{\circ}\right.$ $03^{\prime} 48^{\prime \prime}$ W) at depth $807.7 \mathrm{~m}$

DE-52. Well 3S/14E-28dada

$30,400 \pm 1000$

Sample coll Sept 6, 1979, from Pennington Co $\left(43^{\circ} 45^{\prime} 10^{\prime \prime}\right.$ N, $102^{\circ}$ $26^{\prime} 31^{\prime \prime} \mathrm{W}$ ) at depth $865.6 \mathrm{~m}$.

\section{E. Wyoming}

DE-53. Well 15N/18W-9bba

$14,200 \pm 350$

Sample coll Aug 9, 1979, from Niobrara Co $\left(\begin{array}{ll}47^{\circ} 05^{\prime} 00^{\prime \prime} & \mathrm{N}, 109^{\circ}\end{array}\right.$ $27^{\prime} 41^{\prime \prime} \mathrm{W}$ ) at depth $187.1 \mathrm{~m}$.

\section{DE-54. Well 16N/17W-18bdd}

$>40,000$

Sample coll Aug 11, 1979, from Niobrara Co $\left(47^{\circ} 09^{\prime} 05^{\prime \prime} \mathrm{N}, 109^{\circ}\right.$ $\left.37^{\prime} 16^{\prime \prime} \mathrm{W}\right)$ at depth $378 \mathrm{~m}$.

\section{DE-55. Well 10N/17W-16bb2}

$17,500 \pm 370$

Sample coll Aug 6, 1979, from Weston Co $\left(47^{\circ} 14^{\prime} 30^{\prime \prime} \mathrm{N}, 110^{\circ} 27^{\prime}\right.$ $52 "$ W) at depth $134.7 \mathrm{~m}$.

DE-56. Well 17N/10W-26bb

$8900 \pm 160$

Sample coll Aug 6, 1979, from Weston Co $\left(47^{\circ} 12^{\prime} 41^{\prime \prime} \mathrm{N}, 110^{\circ} 25^{\prime}\right.$ $24 " \mathrm{~W}$ ) at depth $106.7 \mathrm{~m}$. 
Sample coll Aug 12, 1979, from Niobrara Co $\left(47^{\circ} 11^{\prime} 36^{\prime \prime} \mathrm{N}, 109^{\circ}\right.$ $48^{\prime} 06^{\prime \prime} \mathrm{W}$ ) at depth 507.5m.

DE-58. Well 16N/17W-36aca

$33,500 \pm 1900$

Sample coll Aug 12, 1979, from Niobrara Co $\left(47^{\circ} 06^{\prime} 33^{\prime \prime}\right.$ N, $109^{\circ}$ $30^{\prime} 32^{\prime \prime} \mathrm{W}$ ) at depth $118.9 \mathrm{~m}$.

DE-59. Well 14N/18W-10dbb

Sample coll Aug 9, 1979, from Niobrara Co $\left(45^{\circ} 59^{\prime} 11^{\prime \prime} \mathrm{N}, 109^{\circ}\right.$ $04^{\prime} 13^{\prime \prime}$ W) at depth $28 \mathrm{~m}$.

DE-60. Well 16N/17W-2ladd

Sample coll Aug 12, 1979, from Niobrara Co $\left(47^{\circ} 07^{\prime} 54^{\prime \prime} \mathrm{N}, 109^{\circ}\right.$ $34^{\prime} 06^{\prime \prime} \mathrm{W}$ ) at depth $88.4 \mathrm{~m}$.

\section{DE-61. Well 18N/10W-35cdl}

Sample coll Aug 8, 1979, from Weston Co $\left(47^{\circ} 16^{\prime} 32^{\prime \prime} \mathrm{N}, 110^{\circ} 25^{\prime}\right.$ $04^{\prime \prime} \mathrm{W}$ ) at depth $247.3 \mathrm{~m}$.

DE-62. Well 15N/18W-27aa

$$
4200 \pm 140
$$

Sample coll Aug 9, 1979, from Niobrara Co $\left(47^{\circ} 02^{\prime} 19^{\prime \prime} \mathrm{N}, 109^{\circ} 25^{\prime}\right.$ $\left.14^{\prime \prime} \mathrm{W}\right)$ at depth $152.4 \mathrm{~m}$. Comment: possible contamination from underlying strata.

DE-63. Well 16N/17W-28ade

$>\mathbf{3 9 , 2 0 0}$

Sample coll Aug 11, 1979, from Niobrara Co $\left(47^{\circ} 07^{\prime} 21^{\prime \prime} \mathrm{N}, 109^{\circ}\right.$ $04^{\prime} 13^{\prime \prime}$ W) at depth $221 \mathrm{~m}$.

DE-64. Well 16N/18W-32ded

$18,600 \pm 280$

Sample coll Aug 11, 1979, from Niobrara Co $\left(47^{\circ} 05^{\prime} 58^{\prime \prime} \mathrm{N}, 109^{\circ}\right.$ $27^{\prime} 58^{\prime \prime} \mathrm{W}$ ) at depth $213.4 \mathrm{~m}$.

DE-65. Well 17N/10W-20bd $10,100 \pm 180$

Sample coll Aug 7, 1979, from Weston Co $\left(47^{\circ} 13^{\prime} 13^{\prime \prime} \mathrm{N}, 110^{\circ} 29^{\prime}\right.$ $00^{\prime \prime} \mathrm{W}$ ) at depth $33.5 \mathrm{~m}$.

DE-66. Well 16N/10W-5aa $\quad 8100 \pm 180$ Sample coll Aug 7, 1979, from Weston Co $\left(47^{\circ} 11^{\prime} 02^{\prime \prime} \mathrm{N}, 110^{\circ} 27^{\prime}\right.$ $37^{\prime \prime} \mathrm{W}$ ) at depth $14 \mathrm{~m}$.

DE-67. Well 18N/11W-22ce >36,000 Sample coll Aug 4, 1979, from Weston Co $\left(47^{\circ} 18^{\prime} 11^{\prime \prime} \mathrm{N}, 105^{\circ} 19^{\prime}\right.$ $08^{\prime \prime} \mathrm{W}$ ) at depth $345.6 \mathrm{~m}$.

DE-68. Well 18N/10W-13ca

$33,300 \pm 2100$

Sample coll Aug 4, 1979, from Weston Co (47 $18^{\prime} 49^{\prime \prime} \mathrm{N}, 110^{\circ} 23^{\prime}$ $47^{\prime \prime} \mathrm{W}$ ) at depth $422.8 \mathrm{~m}$. 


\section{REFERENCES}

Brown, Eugene, Skougstad, M W, and Fishman, M J, 1970, US Geological Survey techniques of water resources investigations: Book 5 , Chap Al, p 42-44.

Calf, G E and Polach, H A, 1974, Teflon vials for liquid scintillation counting of carbon-14 samples, in Stanley, P E and Scoggins, B A, eds, Internatl symposium on liquid scintillation counting, recent developments: New York, Academic Press, p 223.

Craig, Harmon, 1957, Isotopic standards for carbon and oxygen and factors for massspectrometric analysis of carbon dioxide: Geochim et Cosmochim Acta, v 12, p 133-149.

Noakes, J E, Kim, S M, and Akers, L K, 1967, Recent improvements in benzene chemistry for radiocarbon counting: Geochim et Cosmochim Acta, v 31, p 10941096.

Schroder, L J, Beetem, W A, Claassen, H C, and Emerson, R L, 1973, US Geological Survey, Denver, Colorado radiocarbon dates I: Radiocarbon, v 15, p 469-478.

Schroder, L J, Emerson, R L, and Beetem, W A, 1978, US Geological Survey, Denver, Colorado radiocarbon dates II: Radiocarbon, v 20, p 200-209.

Yang, I C and Emerson, R L, 1980, Teflon vials for low-level C-14 liquid scintillation counting, in Peng, C 'T, Horrocks, D L, and Alpen, E L, eds, Internatl conf on liquid scintillation counting, recent applications and developments: New York, Academic Press, v 2, p 181-197. 